\title{
Remarks on Multi-Dimensional Conformal Mechanics ${ }^{\star}$
}

Čestmír BURDÍK ${ }^{\dagger}$ and Armen NERSESSIAN $\ddagger \S$

${ }^{\dagger}$ FNSPE, Czech Technical University in Prague Trojanova 13, 12000 Prague 2, Czech Republic E-mail: burdik@kmlinux.fjfi.cvut.cz

¥ Artsakh State University, 5 M. Gosh Str., Stepanakert, Armenia

$\S$ Yerevan State University, 1 A. Manoogian Str., 0025, Yerevan, Armenia

E-mail: arnerses@yerphi.am

Received October 30, 2008, in final form January 10, 2009; Published online January 12, 2009 doi:10.3842/SIGMA.2009.004

Abstract. Recently, Galajinsky, Lechtenfeld and Polovnikov proposed an elegant grouptheoretical transformation of the generic conformal-invariant mechanics to the free one. Considering the classical counterpart of this transformation, we relate this transformation with the Weil model of Lobachewsky space.

Key words: conformal mechanics; integrability

2000 Mathematics Subject Classification: 70H33; 70H06

Since the middle of the seventies, after [1], much attention paid, in the field-theoretical literature, to the simple one-dimensional mechanical system given by the Hamiltonian

$$
H=\frac{p^{2}}{2}+\frac{g^{2}}{2 x^{2}} .
$$

The reason was that this Hamiltonian, together with the generators

$$
D=p x, \quad K=\frac{x^{2}}{2}
$$

forms the conformal algebra $s o(1,2)$ with respect to the canonical Poisson brackets $\{p, x\}=1$ :

$$
\{H, D\}=2 H, \quad\{H, K\}=D, \quad\{K, D\}=-2 K .
$$

Here $D$ is the dilatation and $K$ is the conformal boost. It was also observed, that the action functional of this system is invariant under conformal transformations, up to total derivative term. Thanks to this observation the above system presently known in literature as "one-dimensional conformal mechanics". Notice, however, that this system is conformal invariant in the fieldtheoretical context only, since its action functional possesses a conformal symmetry provided that the time reparametrizations are admitted. While the Hamiltonian (1) is not invariant under conformal transformation, and the generators $D, K$ do not constitute the conserved quantities, see (3). The matter is that in classical mechanics the invariance transformations do not admit the time reparametrizations. This seeming contradiction can be explained as follows: to admit the time reparametrizations, the initial nondegenerated Lagrangian should be replaced by the degenerated reparametrization-invariant one. The former Lagrangian defines unconstrained Hamiltonian system with two-dimensional phase space, and the latter one defines the constrained Hamiltonian system with four-dimensional phase space, where the role of additional coordinate plays the time parameter, and the constraint plays the role of Hamiltonian. The latter system

${ }^{\star}$ This paper is a contribution to the Proceedings of the XVIIth International Colloquium on Integrable Systems and Quantum Symmetries (June 19-22, 2008, Prague, Czech Republic). The full collection is available at http://www.emis.de/journals/SIGMA/ISQS2008.html 
is invariant under conformal transformations, given by the above generators properly extended by the time-dependent terms. Reducing the system to the nondegenerate one, we arrive to the above presented one-dimensional "conformal mechanics". For the higher-dimensional mechanical systems the conformal invariance the same explanation.

One-dimensional conformal mechanics (1) can be considered as a two-particle Calogero model (with the excluded center of mass), one-dimensional multi-particle integrable system with inverse-square interaction [2]

$$
\mathcal{H}_{\text {Calogero }}=\sum_{i=1}^{d+1} \frac{p_{i}^{2}}{2}+\sum_{i<j=1}^{d+1} \frac{g^{2}}{\left(x^{i}-x^{j}\right)^{2}}, \quad\left\{p_{i}, q^{j}\right\}=\delta_{i}^{j} .
$$

The quantum Calogero model is defined by the above Hamiltonian, where the classical momenta $p_{i}$ are replaced by the respective operators $\widehat{p}_{i}=-\imath \partial / \partial x^{i}$, and the coupling constant $g^{2}$ is replaced by $g(g-1)$. This model has attracted much attention due to numerous applications in a wide area of physics, as well as due to a rich internal structure (see, e.g., the recent review [3] and references therein). Already in the pioneering paper [2] it was observed that the spectrum of the Calogero model with an additional oscillator potential is similar to the spectrum of free $N$-dimensional oscillator. It was claimed there that a similarity transformation to the free oscillator system may exist, at least, in part of the Hilbert space. However, this transformation was written explicitly only decades after [4]. Later on Galaginsky, Lechtenfeld and Polovnikov found the unitary transformation, defined by the $S U(1,1)$ group element [5], which maps the Hamiltonian of the arbitrary conformal mechanics in the Hamiltonian of free particle (further we shall refer it as GLP-transformation). This observation allowed them to build not only the $\mathcal{N}=2$ supersymmetric Calogero model [6] but the $\mathcal{N}=4$ superconformal Calogero model as well [7]. In [8] GLP-transformation was extended to the higher-dimensional many-body systems with conformal Galilean symmetry. So any conformal mechanics can be mapped on to the free system but the prize is the nonlocality of transformation suggested in [5]. An important and nontrivial feature of the GLP-transformation is the presence of quantum corrections in the transformed generator of conformal boosts: these corrections provide the possibility to built $\mathcal{N}=4$ superconformal Calogero.

Recently, one of the authors (A.N.), in collaboration with T. Hakobyan, proposed a simple classical analog of the decoupling transformation for the one-dimensional conformal mechanics (1) (and for its $\mathcal{N}=2 k$ superconformal extension) [9]. Namely, they parameterized the phase space of the one-dimensional conformal mechanics by the single complex coordinate

$$
w=\frac{p}{x}+\frac{\imath g}{x^{2}}, \quad \operatorname{Im} w>0: \quad\{w, \bar{w}\}=-\frac{\imath}{g}(w-\bar{w})^{2} .
$$

In other words, the phase space of the system was identified with the Klein model of the Lobachevsky plane.

In this parametrization, the $s o(1,2)$ generators $(1),(2)$ define the Killing potentials (Hamiltonian generators of the isometries of the Kähler structure) of the Lobachevsky plane given by the following metric and potential

$$
d s^{2}=-\frac{g d w d \bar{w}}{(\bar{w}-w)^{2}}, \quad \mathcal{K}=g \log \imath(\bar{w}-w) .
$$

Explicitly,

$$
H=\imath g \frac{w \bar{w}}{w-\bar{w}}, \quad D=\imath g \frac{w+\bar{w}}{w-\bar{w}}, \quad K=\imath g \frac{1}{w-\bar{w}} .
$$

The Kähler structure (5) is invariant under the discrete transformation

$$
w \rightarrow-\frac{1}{w}
$$


whereas the Killing potentials (6) transform s follows:

$$
H \rightarrow K, \quad K \rightarrow H, \quad D \rightarrow-D .
$$

So in the one-dimensional case everything is nice and local. However hard to believe, this transformation can be extended to the $d>1$-dimensional conformal mechanics in a local (i.e. canonical) way. The discussion of this and related issues is the purpose of the present note.

For this purpose we, at first, introduce an appropriate "radial" coordinate and conjugated momentum, so that the higher-dimensional system looks very similar to the one-dimensional conformal mechanics. In that picture, the rest ("angular") of the degrees of freedom are packed in the Hamiltonian system on the $(d-1)$-dimensional sphere: it replaces the coupling constant $g^{2}$ in the one-dimensional conformal mechanics, and defines the constant of motion of the is initial conformal mechanics. Exploiting this observation we build, by the use of textbook integrable systems on the spheres, some examples of integrable conformal mechanics. Then we relate, the radial part of the $d$-dimensional conformal mechanics with the Klein model of the Lobachevsky space, which is completely similar to the above-presented one-dimensional case. However we find, that an analog of (7) transformation necessarily changes the "angular part" of the Poisson brackets of the decoupling, i.e., is noncanonical one.

Let us consider the $d$-dimensional conformal mechanics defined by the following Hamiltonian and symplectic structure:

$$
\omega=d \mathbf{p} \wedge d \mathbf{r}, \quad \mathcal{H}=\frac{\mathbf{p}^{2}}{2}+V(\mathbf{r}), \quad \text { where } \quad \mathbf{r} \cdot \nabla V(\mathbf{r})=-2 V(\mathbf{r}) .
$$

This Hamiltonian together with the generators

$$
D=\mathbf{p} \cdot \mathbf{r}, \quad K=\frac{\mathbf{r}^{2}}{2}
$$

forms the conformal algebra so(1,2) (3). Here $D$ defines the dilatation and $K$ defines the conformal boost, $\mathbf{r}=\left(x^{1}, \ldots, x^{d}\right), \mathbf{p}=\left(p_{1}, \ldots, p_{d}\right)$, and the Poisson brackets are assumed to be canonical $\left\{p_{i}, x^{j}\right\}=\delta_{i}^{j}$.

Extracting the radius $r=|\mathbf{r}|$ we can represent the above generators in the following form:

$$
\mathcal{D}=p_{r} r, \quad \mathcal{K}=\frac{r^{2}}{2}, \quad \mathcal{H}=\frac{p_{r}^{2}}{2}+\frac{I}{r^{2}}, \quad I \equiv \frac{\mathbf{J}^{2}}{2}+U, \quad U \equiv r^{2} V(\mathbf{r}) .
$$

Here $p_{r}=\mathbf{p r} / r$ is the momentum conjugated to the radius $\left\{p_{r}, r\right\}=1$.

It is seen that the function $U$ obeys the condition

$$
\mathbf{r} \cdot \nabla U=0 .
$$

Introducing some spherical coordinates $\phi^{\alpha}$ and their canonically conjugated momenta $\pi_{\alpha}$, we get, that the function $U$ is independent of the radius

$$
\frac{\partial U}{\partial r}=0
$$

So, the function $I(u), u^{a}=\left(\pi_{\alpha}, \phi^{\alpha}\right)$ defines the constant of motion of the system

$$
\{\mathcal{H}, I(u)\}=0 .
$$

Hence, the conserved quantity $I$ defines the motion of particle on the $(d-1)$-dimensional sphere, in the presence of potential $U(\phi)$, so that its phase space is the cotangent bundle of $(d-1)$ dimensional sphere, $T^{*} S^{d-1}$. Parameterizing $T^{*} S^{d-1}$ by the canonical variables $u^{a}$, we can represent this Hamiltonian system in the form

$$
\left(T^{*} S^{d-1}, \omega_{0}=d \pi_{\alpha} \wedge d \phi^{\alpha}, I(u)=\frac{1}{2} g^{\alpha \beta}(\phi) \pi_{\alpha} \pi_{\beta}+U(\phi)\right) .
$$


where $g^{\alpha \beta}(\phi)$ is the inverse metrics of the $(d-1)$-dimensional sphere. It is clear, that the integrable $d$-dimensional conformal mechanics defines the integrable $(d-1)$-dimensional system on the sphere, and vice versa. It deserves to be mentioned that the generators of conformal algebra obey the equality:

$$
4 \mathcal{H} \mathcal{K}-\mathcal{D}^{2}=2 I
$$

Let us try to solve the equations of motion of the conformal mechanics in the parametrization given by (8) and (9). Keeping in mind that $p_{r}=\dot{r}$ and fixing the values of the constants of motion $\mathcal{H}=E$ and $I=I_{0}$, we can immediately find the evolution of $r$,

$$
t=\int \frac{d r}{\sqrt{2 E-\frac{2 I_{0}}{r^{2}}}} \Rightarrow r^{2}= \begin{cases}2 E t^{2}+\frac{I_{0}}{E} & \text { for } E \neq 0, \\ 2 \sqrt{-2 I_{0}} t & \text { for } E=0\end{cases}
$$

This procedure is completely to the one performed in the Landau and Lifshits textbook on classical mechanics [10] for the one-dimensional systems and for the three-dimensional spherically symmetric systems. The analyzes of the dynamics of the presented effective system on from the initial conditions is also similar to those presented in [10]. Particulary, for $I_{0}>0$ we get the one-dimensional system with effectively repulsive force, and the energy $E$ takes positive values only. The case $I_{0}<0$ leads to the one-dimensional system with with the "effectively attractive force", and the energy could takes both positive and negative values. In the latter case the range of time could be finite, with corresponds to the "falling on the center" phenomenon.

The evolution of the angular part of the system is defined by the equations

$$
\frac{d u^{a}}{d t}=\left\{u^{a}, \mathcal{H}\right\}=\frac{\left\{u^{a}, I(u)\right\}}{r^{2}(t)} \Rightarrow \frac{d u^{a}}{d T}=\left\{u^{a}, I\right\},
$$

where $T$ is the new evolution parameter

$$
T=T(t) \equiv \int \frac{d t}{r^{2}(t)}
$$

Hence, supposing that (9) is the integrable system and we have the solutions of its equations of motion, $u^{a}=F^{a}(T)$, we can immediately write down the equations of motion of the initial conformal mechanics

$$
u^{a}(t)=F^{a}(T(t)) .
$$

So with the appropriate time reparametrization depending on the initial conditions of the system, we can explicitly integrate its equations of motion.

Let us represent some examples of the systems of that sort.

Example 1. Adding an arbitrary constant $\kappa$ to the Hamiltonian of the free particle on $S^{d-1}$ we get the system on $R^{d}$ with the potential

$$
V=\frac{\kappa}{r^{2}}
$$

More generally, since the addition of the constant to the initial Hamiltonian does not change its integrability property, we can conclude that the adding this additional term (11) to the initial conformal mechanics also preserves the integrability property.

The above-presented system is the simplest and most known example of conformal mechanics. Nevertheless, minor modification of the initial system yields less trivial examples of conformal mechanics. 
For example, one can consider the free particle on $S^{2}$ moving in the presence of a constant magnetic field. This system is still $s o(3)$ invariant. The respective three-dimensional system describe the particle moving in the field of the Dirac monopole.

Similarly, the free-particle moving on $S^{4}$ in the presence of the BPST instanton, possesses $s o(5)$ symmetry. The respective five-dimensional conformal mechanics describes the particle on $R^{5}$ interacting with Yang monopole.

Example 2. The $(d-1)$-dimensional spherical (Higgs) oscillator is defined (up to constant) by the potential [11]

$$
V_{\text {Higgs }}=\frac{\omega^{2} \tan ^{2} \theta}{2}
$$

It has $(d-1)^{2}$ constants of motions defining the nonlinear deformation of $s u(d-1)$ algebra. The corresponding conformal mechanics is given by the potential

$$
V_{\mathrm{conf}}=\frac{\omega^{2}}{2 x_{d}^{2}}+\frac{\omega^{2}}{2 r^{2}}
$$

and has $(d-1)^{2}+1$ constants of motion. Note that this system loses its exact solvability property in the presence of the monopole (for $d=3$ ) and BPST instanton (for $d=5$ ) fields.

Example 3. The $(d-1)$-dimensional spherical Coulomb system given by the potential $V_{\text {Sch-Coul }}=$ $\gamma \cot \theta$ was suggested by Schrödinger [12]. It has $(d-2)(d-3) / 2$ constants of motion defining the nonlinear deformation of the symmetry algebra of the Coulomb system. So the respective $d$-dimensional conformal mechanics has $(d-2)(d-3) / 2+1$ constants of motion and is given by the potential

$$
V=\gamma \frac{x_{d}}{r^{2} \sqrt{r^{2}-x_{d}^{2}}}
$$

Let us mention the existence of the integrable spherical analogs of the two-center Coulomb system [13] of the anisotropic oscillator, as well as the integrable spherical analog of the Coulomb system in the constant electric field [14]. By the use of these systems we can construct the respective integrable conformal mechanical models.

Example 4. The $(d+1)$-particle Calogero model defined by the Hamiltonian (4) can also be represented in the proposed form. Since this system is translation-invariant, one can transit to the center-of mass- coordinate frame, reducing the initial $d$-dimensional system to the $(d-1)$ dimensional one. The two-particle Calogero model results, upon excluding the center of mass, in the one-dimensional conformal mechanics given by Hamiltonian (1). In the three- and more particle case the resulted system is more complicated. Splitting its radial and angular parts we get the integrable system on the $(d-1)$-dimensional sphere, interacting with the $(d-1)(d-2) / 2$ force centers by the Higgs oscillators law. This centers are located on the sphere in the special order. For example, for the three-particle Calogero system we have three force centers on the circle, with the $2 \pi / 3$ angles between them. The four-particle Calogero model yields the system on the two dimensional sphere, with the force centers located at the vertices of cuboctahedron [15].

Let us also mention the recent paper [16], where the representation (8) has been used for the construction of $\mathcal{N}=4$ superconformal extension of the three-dimensional conformal mechanics with the arbitrary positive function $U(u)$, including, as a particular cases, the tree-particle Calogero models of various types. 
Now let us consider the analog of decoupling transformation of one-dimensional conformal mechanics, given by (7) in the higher-dimensional case. We shall use the representation of conformal mechanics given by (8). As we have already mentioned, the conserved quantity $I^{2}$ defines the motion of particle on the $(d-1)$-dimensional sphere in the presence of the potential $U$, so that its phase space is the cotangent bundle of $(d-1)$-dimensional sphere, $T^{*} S^{d-1}$. Parameterizing $T^{*} S^{d-1}$ by the canonical variables $u^{a}=\left(\pi_{\alpha}, \phi^{\alpha}\right)$, we can represent this Hamiltonian system in the form given by (9).

Similarly to the one-dimensional case [9], we introduce, instead of the radius $r$ and its conjugated momentum $p_{r}$, the following complex variable (we restrict ourselves, for simplicity, to a particular case $I>1)$ :

$$
w=\frac{p_{r}}{r}+\frac{\imath \sqrt{2 I}}{r^{2}} \equiv \frac{\mathcal{D}+\imath \sqrt{2 I}}{2 \mathcal{K}}, \quad \operatorname{Im} w>0 .
$$

This complex variable obeys the following Poisson bracket relation

$$
\{w, \bar{w}\}=-\frac{\imath}{\sqrt{2 I(u)}}(w-\bar{w})^{2} .
$$

Taking into account equality (10) we can write

$$
\mathcal{H}=\imath \sqrt{2 I(u)} \frac{w \bar{w}}{w-\bar{w}}, \quad \mathcal{D}=\imath \sqrt{2 I(u)} \frac{w+\bar{w}}{w-\bar{w}}, \quad \mathcal{K}=\imath \frac{\sqrt{2 I(u)}}{w-\bar{w}},
$$

Now, completely similarly to the one-dimensional case, described in the introduction, we can define the decoupling transformation

$$
w \rightarrow-\frac{1}{w}: \quad \mathcal{H}=\frac{p_{r}^{2}}{2}+\frac{I}{r^{2}} \quad \rightarrow \quad \mathcal{K}=\frac{r^{2}}{2},
$$

and $\mathcal{K} \rightarrow-\mathcal{H}$. Keeping in mind the expressions (8), let us represent the transformed coordinate $\widetilde{w}$ as follows

$$
\widetilde{w} \equiv-\frac{\widetilde{r}}{\widetilde{p}_{r}}+\imath \frac{\sqrt{2 I(u)}}{\widetilde{p}_{r}^{2}} .
$$

In that case, the transformation (14) yields the following transformation of the radius and conjugated momentum. In the initial coordinates this transformation looks like

$$
\widetilde{p}_{r}= \pm \sqrt{p_{r}^{2}+2 I / r^{2}}=\sqrt{2 \mathcal{H}}, \quad \widetilde{r}= \pm \frac{p_{r} r}{\sqrt{p_{r}^{2}+2 I / r^{2}}}= \pm \frac{\mathcal{D}}{2 \sqrt{\mathcal{H}}} .
$$

Hence, everything looks completely similar to the one-dimensional case.

Let us notice that the transformed Hamiltonian $\mathcal{K}$ does not contain any information on the specific system. Also, the obtained transformation (15) can be written without refereeing to the internal structure of the Hamiltonian. The only additional constraint is given by (10). The matter is that in our representation the essential information on the system is encoded in the Poisson brackets which depend on the "spherical" Hamiltonian $I$ and is explicitly defined by the use of the equations of motion. Indeed, the Poisson brackets are defined not only by relation (12), but also by the following ones:

$$
\left\{u^{a}, u^{b}\right\}=\omega^{a b}(u), \quad\left\{u^{a}, w\right\}=(w-\bar{w}) \frac{V^{a}(u)}{2 I}, \quad\left\{u^{a}, \bar{w}\right\}=(w-\bar{w}) \frac{V^{a}(u)}{2 I},
$$

where $V^{a}=\left\{u^{a}, I(u)\right\}$ are the equations of motion of the spherical system (9), and $\omega^{a b}(u)$ are its the Poisson brackets. 
The symplectic structure of the conformal mechanics can be represented as follows:

$$
\Omega=-\imath \frac{\sqrt{2 I(u)} d w \wedge d \bar{w}}{(\bar{w}-w)^{2}}+\frac{(d w+d \bar{w}) \wedge d \sqrt{2 I(u)}}{\imath(\bar{w}-w)}+\frac{1}{2} \omega_{a b} d u^{a} \wedge d u^{b}
$$

while the local one-form defining this symplectic structure reads

$$
\Omega=d \mathcal{A}, \quad \mathcal{A}=\imath \sqrt{2 I(u)} \frac{d w+d \bar{w}}{\imath(w-\bar{w})}+A_{0}(u), \quad d A_{0}=\frac{1}{2} \omega_{a b} d u^{a} \wedge d u^{b} .
$$

It is convenient to represent these forms as follows:

$$
\begin{aligned}
& \Omega=-\imath \frac{\sqrt{2 I(u)} d w \wedge d \bar{w}}{(\bar{w}-w)^{2}}-\imath \frac{w+\bar{w}}{\bar{w}-w} d \log (w+\bar{w}) \wedge d \sqrt{2 I(u)}+\frac{1}{2} \omega_{a b} d u^{a} \wedge d u^{b}, \\
& \mathcal{A}=-\imath \sqrt{2 I(u)} \frac{w+\bar{w}}{\bar{w}-w} d \log (w+\bar{w})+A_{0}(u) .
\end{aligned}
$$

It is seen that transformation (14) does not preserves the symplectic structure, i.e., it is not a canonical transformation for the generic $d>1$-dimensional conformal mechanics. To make the transformation canonical, we have to admit the time reparametrizations and to mix the "angular" coordinates $u^{a}$ with the "radial" one given by $w$. This means, that we can't make the decoupling transformation canonical in the strong sense (assuming preservation of the symplectic structure $d \mathbf{p} \wedge d \mathbf{r}$ ). But the decoupling transformation could be extended to the "false" canonical transformation (in the sense of [10]), which assumes the preservation of the contact structure $\mathbf{p} d \mathbf{r}-\mathcal{H} d t$ and, consequently, of the Hamiltonian equations of motion. The discussion of the difference between these definitions can be found in [17].

Remark. Considering the decoupling transformation of conformal mechanics, we restricted ourself by the particular case $I>0$. For the $I<0$ case the discussion and conclusions are very similar former one. We should simply replace $\imath \sqrt{2 I}$ by $\sqrt{-2 I}$. In that case $w$ and $\bar{w}$ become real variables, and the symplectic structure can not be related with the Kähler structure. Consequently, the generators (13) can not be interpreted as a Killing potentials.

Straightforward transition to the quantum mechanics via canonical quantization is ill defined procedure for the presented picture. The relevant quantization scheme for the one-dimensional conformal mechanics is the geometric quantization (which is well-defined procedure for the quantization on Lobachevsky space). Even in this simplest case on quantum mechanical level we shall get some new peculiarities, when consider the decoupling transformation. Namely, the inversion procedure would change the range of validity of the wavefunction. In other worlds, it will define the unitary transformation for the part of the Hilbert space of the initial system only. One can expect, that in the $d>1$ dimensional case we must deal similar to the one-dimensional case, with the radial part, encoded in $w$ coordinate. On the other hand in in our construction the main role is given to the square root from the "spherical" Hamiltonian, $\sqrt{I}$. But taking the "square root" from the quantum-mechanical operator is dangerous. One can, seemingly, to avoid it for the class of quantum spherical Hamiltonians, which are factorisable ones. In that case the operator $\sqrt{I}$ will be just the linear operator in the canonically quantized system. This will yields, obviously, nontrivial quantum corrections, observed in [5].

\section{Acknowledgments}

The work is done in accordance with the research plan of the Czech Ministry of Education MSM6840770039, and partially supported by grant of the Artsakh Ministry of Education and Science and by NFSAT-CRDF UC 06/07 and INTAS-05-7928 grants. 


\section{References}

[1] de Alfaro V., Fubini S., Furlan G., Conformal invariance in quantum mechanics, Nuovo Cimento A 34 (1976), 569-612.

[2] Calogero F., Solution of a three-body problem in one-dimension, J. Math. Phys. 10 (1969), 2191-2196. Calogero F., Solution of the one-dimensional $N$-body problems with quadratic and/or inversely quadratic pair potentials, J. Math. Phys. 12 (1971), 419-436.

[3] Polychronakos A.P., Physics and mathematics of Calogero particles, J. Phys. A: Math. Gen. 39 (2006), 12793-12845, hep-th/0607033.

[4] Gurappa N., Prasanta K., Equivalence of the Calogero-Sutherland model to free harmonic oscillators, Phys. Rev. B 59 (1999), R2490-R2493, cond-mat/9710035.

Ghosh P.K., Super-Calogero-Moser-Sutherland systems and free super-oscillators: a mapping, Nuclear Phys. B 595 (2001), 519-535, hep-th/0007208.

Brzezinski T., Gonera C., Maslanka P., On the equivalence of the rational Calogero-Moser system to free particles, Phys. Lett. A 254 (1999), 185-196, hep-th/9810176.

[5] Galajinsky A., Lechtenfeld O., Polovnikov K., Calogero models and nonlocal conformal transformations, Phys. Lett. B 643 (2006), 221-227, hep-th/0607215.

[6] Freedman D.Z., Mende P.F., An exactly solvable $N$-particle system in supersymmetric quantum mechanics, Nuclear Phys. B 344 (1990), 317-343.

[7] Galajinsky A., Lechtenfeld O., Polovnikov K., $N=4$ superconformal Calogero models, J. High Energy Phys. 2007 (2007), no. 11, 008, 23 pages, arXiv:0708.1075.

Galajinsky A., Lechtenfeld O., Polovnikov K., $N=4$ mechanics, WDVV equations and roots, arXiv:0802.4386.

Lechtenfeld O., WDVV solutions from orthocentric polytopes and Veselov systems, arXiv:0805.3245.

[8] Galajinsky A.V., Remark on quantum mechanics with conformal Galilean symmetry, Phys. Rev. D 78 (2008), 087701, 3 pages, arXiv:0808.1553.

[9] Hakobyan T., Nersessian A., Lobachevsky geometry of (super)conformal mechanics, arXiv:0803.1293.

[10] Landau L.D., Lifshits E.M., Mechanics, 5th ed., Nauka, Moscow, 2004.

[11] Higgs P.W., Dynamical symmetries in a spherical geometry. I, J. Phys. A: Math. Gen. 12 (1979), 309-323. Leemon H.I., Dynamical symmetries in a spherical geometry. II, J. Phys. A: Math. Gen. 12 (1979), 489-501.

[12] Schrödinger E., A method of determining quantum-mechanical eigenvalues and eigenfunctions, Proc. Roy. Irish Acad. Sect. A 46 (1941), 9-16.

Schrödinger E., Further studies on solving eigenvalue problems by factorization, Proc. Roy. Irish Acad. Sect. A 46 (1941), 183-206.

Schrödinger E., The factorization of the hypergeometric equation, Proc. Roy. Irish Acad. Sect. A 47 (1941), 53-54, physics/9910003.

[13] Otchik V.S., Symmetry and separation of variables in the two-center Coulomb problem in three dimensional spaces of constant curvature, Dokl. Akad. Nauk BSSR 35 (1991), 420-424 (in Russian).

[14] Nersessian A., Yeghikyan V., Anisotropic inharmonic Higgs oscillator and related (MICZ-)Kepler-like systems, J. Phys. A: Math. Theor. 41 (2008), 155203, 11 pages, arXiv:0710.5001.

[15] Hakobyan T., Nersessian A., Yeghikyan V., Cuboctahedric Higgs oscillator from the Calogero model, arXiv:0808.0430.

[16] Bellucci S., Krivonos S., Sutulin A., $N=4$ supersymmetric 3-particles Calogero model, Nuclear Phys. B 805 (2008), 24-39, arXiv:0805.3480.

[17] Arnold V.I., Mathematical methods in classical mechanics, Nauka, Moscow, 1973. 